\title{
Conformal Mapping of Impedance Stability Models for System-Level Dynamics As-
} sessments

F. Freijedo, U. Javaid, and D. Dujic

This material is posted here with permission of the IEEE. Such permission of the IEEE does not in any way imply IEEE endorsement of any of EPFL's products or services. Internal or personal use of this material is permitted. However, permission to reprint / republish this material for advertising or promotional purposes or for creating new collective works for resale or redistribution must be obtained from the IEEE by writing to pubs-permissions@ieee. org. By choosing to view this document, you agree to all provisions of the copyright laws protecting it. 


\title{
Conformal Mapping of Impedance Stability Models for System-Level Dynamics Assessments
}

\author{
Francisco D. Freijedo, Uzair Javaid and Drazen Dujic \\ Power Electronics Laboratory \\ Ecole Polytechnique Federale de Lausanne \\ CH-1015, Lausanne, Switzerland. \\ E-mails: \{francisco.freijedo, uzair.javaid, drazen.dujic\}@epfl.ch.
}

\begin{abstract}
Impedance stability methods are suitable for assessing the dynamics of power converters controllers, but also for ac/dc microgrids system level studies. This work proposes the combination of impedance modeling and conformal mapping for the identification of the dominant eigenvalues. The methodology is derived by linearization of the Cauchy-Riemann equations, which define the conformal mapping property of the Nyquist trajectories. The proposed technique is of high practical value, since it can be performed from frequency-domain data obtained by small-signal perturbation (i.e., explicit transfer functions are not needed). A detailed study case, focused on a medium-voltage dc microgrid, is provided in order to verify the theoretical approach. The new features and limitations of the proposed technique are summarized in the conclusions.
\end{abstract}

Index Terms-ac-dc power conversion, dc power transmission, power system modeling, power system stability, stability criteria.

\section{INTRODUCTION}

The controller design problem based on an impedance/admittance modeling was proposed in classic references [1], [2]. Firstly, a physical system (of any kind) is modelled by an input admittance $Y_{i}(\omega)$; then, it is connected to an environment modelled by an output impedance $Z_{o}(\omega)$; finally, the dynamic properties are given by the product $Y_{i}(\omega) Z_{o}(\omega)[1],[2]$.

An analytical approach considers the Laplace variable $s=j \omega$. Then, by assuming that $Y_{i}(s)$ and $Z_{o}(s)$ are analytic, the eigenvalues of the system are given by

$$
1+\underbrace{Y_{o}(s) Z_{i}(s)}_{G(s)}=0
$$

with $G(s)$ being defined as the minor loop gain. An analogy with a classical controller scheme is depicted in Fig. 1. This approach gives an immediate description of system dynamics, which, by some basic design assumptions, can be employed for the design of power electronics controllers [3]. In principle, it is also suitable for an accurate assessment of large power systems [4]. However, since it requires the use of parametric transfer functions, which is hardly feasible in a large systems including power electronics devices (complex controllers, in-

This work is partly funded by the project "Medium-Voltage Direct-Current Energy Conversion Technologies and Systems (SI/501259) granted by the Swiss Federal Office of Energy.

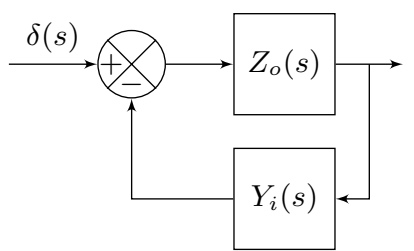

Fig. 1. Simplified representation of the impedance control problem [2].

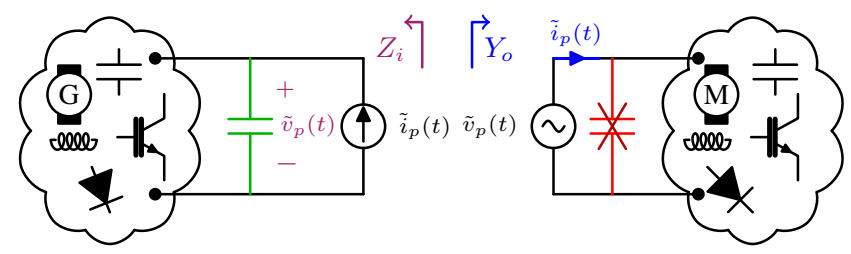

Fig. 2. Impedance modeling of a generic systems.

tellectual property issues), direct calculation of (1) is hardly possible in practice [5].

Alternatively, a data-driven approach considers the shapes of $Y_{i}(\omega)$ and $Z_{o}(\omega)$ for a finite number of $\omega$ values. The descriptions of $Y_{i}(\omega)$ and $Z_{o}(\omega)$ can be obtained by smallsignal perturbation, as shown in Fig. 2. Subsequently, stability is assessed by the Nyquist criterion [5], [6]. This approach has been considered in different applications: ac/dc microgrids [5], [6], renewable energies [7]-[10] and traction [11].

The impedance/admittance modeling has recently gained more and more attention in renewable energy applications, which are characterized by a high penetration of power electronics devices [7]-[10]. From a computational point of view, one main idea behind the impedance/admittance modeling is to avoid too detailed and complex implementations in platforms more focused for large power systems analysis. By means of linearization techniques, Norton/Thevenin equivalents that closely emulate activelly-controlled power converters in the range of frequencies at which the harmonic instability problems are obtained; these linear models can be grouped/lumped and have a low computational burden [7], [9], [10].

A similar methodology is employed in low-voltage/mediumvoltage dc (LVDC/MVDC) systems [6], [12]. The source/load dynamic interactions of a possible MVDC electrical distribution system (see Fig. 3), intended for a large marine vessel 


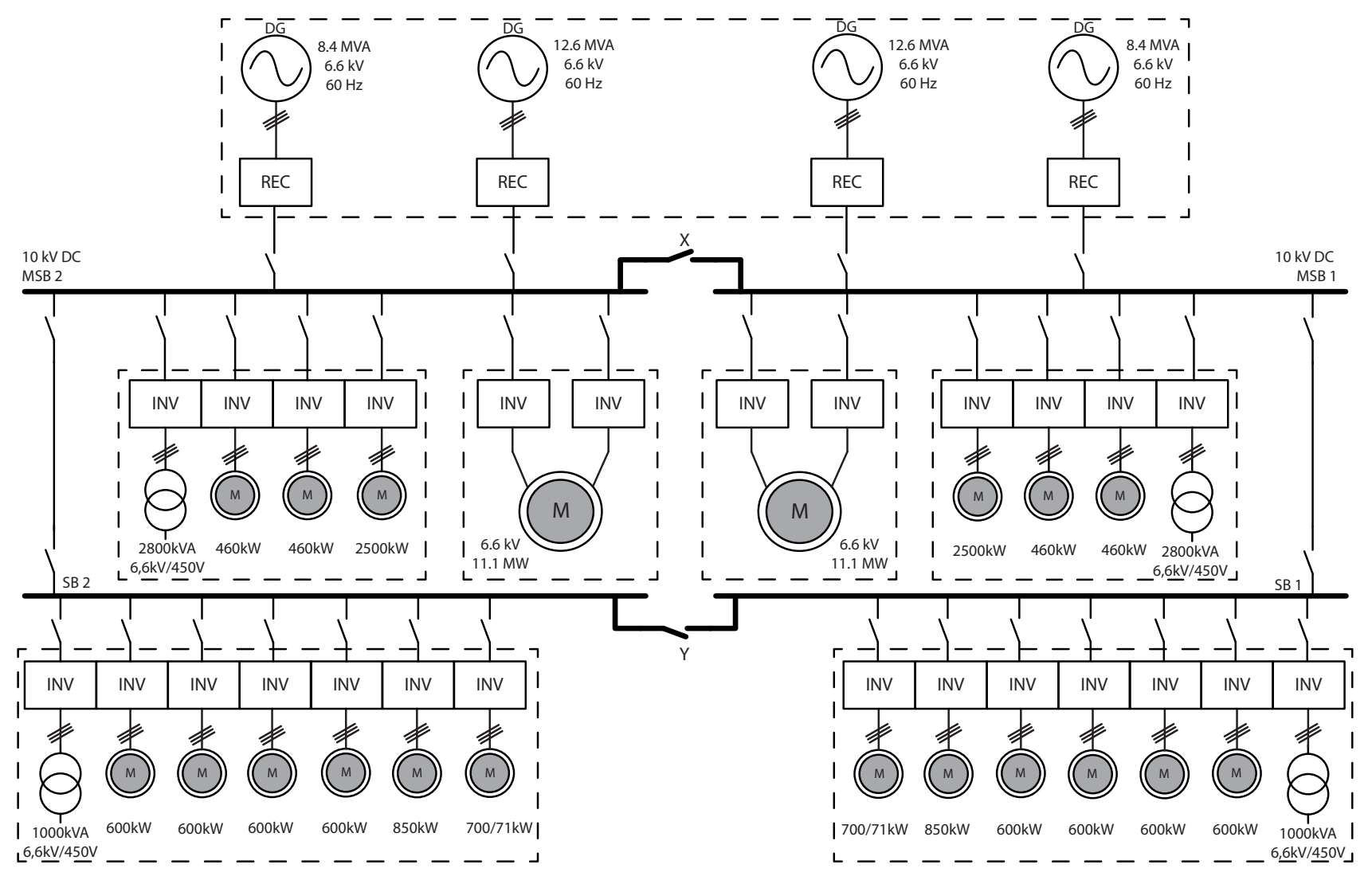

Fig. 3. A possible MVDC power system for ships [12].

with power levels in the range of tens of megawatts, have been addressed in [12]. That analysis considers proven and available industrial technologies that suit the MVDC distribution voltage and power ratings. More specifically, that study includes the contribution of generator inductances, dc-bus capacitors, cable effects, and source/load controllers [e.g., constant power load (CPL) behavior]. The methodology of [12] closely follows the IEEE Std. 1709-2010 guidelines, which implies the combination of small-signal perturbation for impedance modelling with the Nyquist stability criteria in order to provide relative stability assessments [13].

Despite its simplicity, some weak spots are also associated to the impedance modelling and assessment, specially when complex/high-order systems are considered. More specifically, the criteria based on stability regions defined in the polar plot (mathematically, defined as the G-plane below) provide ambiguous interpretations when considering different modelling points (which, in principle, is only a conceptual issue) [5], [6]. In order to make the most information from the polar plot (obtained from modelling at any arbitrary point), this paper proposes a technique that exploits the conformal mapping property inherent to the Nyquist trajectories [14][16]. As shown in this paper, from a correct interpretation of the Nyquist diagram, the dominant eigenvalues that define the closed-loop responses can be identified.

The rest of the paper is organized as follows. Section II shows the proposed technique to identify the dominant eigenvalues. It is based on a linear approximation of the Cauchy-Riemann equations, which define the conformal mapping property. Section III provides a study case for a simplified MVDC power system and validates the accuracy of the proposed technique. Finally, the main findings of the work are summarized in the conclusions.

\section{IDENTIFICATION BY CONFORMAL MAPPING}

The Laplace variable $s$ can be re-defined as $s=\sigma+j \omega$ and its $G$-plane mapping function, i.e., the minor loop gain, as $G(\sigma, \omega)=G_{r e}(\sigma, \omega)+j G_{i m}(\sigma, \omega)$ [14]-[16]. Then, the Nyquist contour employed to get the $G$-plane polar plot is a particular case defined by $s=0 \rightarrow j \omega$ (for positive frequencies) [16]. Absolute stability can be checked by inspection of the polar plots: the system is stable if the trajectories of $G(\sigma+j \omega)$ that cross -1 correspond to stable eigenvalues, a condition that mathematically can be expressed as

$$
\sigma<0 \quad \forall G(\sigma+j \omega)=-1 .
$$

Graphically, this property could be applied for absolute stability tests for any analytically defined $G(s)$, without any explicit consideration on number of -1 encirclements and open-loop right half plane (RHP) poles, as shown in Fig. $4^{1}$.

${ }^{1}$ It should be mentioned that this property is represented for the sake of clarity: in practice, if $G(s)$ is available, the calculation of eigenvalues by (1) is straightforward. 
Since $G(\sigma, \omega)$ is defined as an analytical complex function, it fulfills the Cauchy-Riemann equations [14], given by

$$
\begin{gathered}
\frac{\partial G_{r e}(\sigma, \omega)}{\partial \sigma}=\frac{\partial G_{i m}(\sigma, \omega)}{\partial \omega} \\
\frac{\partial G_{r e}(\sigma, \omega)}{\partial \omega}=-\frac{\partial G_{i m}(\sigma, \omega)}{\partial \sigma} .
\end{gathered}
$$

These expressions define a conformal mapping property, which implies that the local angles in the S-plane are preserved after the G-plane mapping [14]-[16].

An approximation to calculate the eigenvalues of the system can be made as depicted in Fig. 5. Firstly, the sensitivity peak $\eta$ is identified by inspection of the polar plot, which also identifies the imaginary part of the dominant root $\omega_{d}$ [15][18]. Subsequently, by a first order approximation of (3), two solutions for the corresponding real part of the eigenvalue are available [one from (3a) and another from (3b)]

$$
\sigma_{d} \approx \Delta \omega \frac{G_{i m}\left(\omega_{d}\right)}{G_{r e}\left(\omega_{d}\right)-G_{r e}\left(\omega_{d}-\Delta \omega\right)}
$$

and

$$
\sigma_{d} \approx-\Delta \omega \frac{G_{r e}\left(\omega_{d}\right)+1}{G_{i m}\left(\omega_{d}\right)-G_{i m}\left(\omega_{d}-\Delta \omega\right)} .
$$

with $\Delta \omega$ being the frequency step between two consecutive data points. Some practical aspects should be considered:

(1) Equations (4a) and (4b) are linear approximations. By inspection of the trajectory defined by $G\left(0, \omega_{d}\right) \rightarrow G\left(\sigma_{d}, \omega_{d}\right)$ in Fig. 5, it is expected that the linear approximation works better closer to the evaluation point to $-1+j 0$. ie, a higher accuracy is expected for low $\eta$ values. This is in correspondence with references that point to $\eta$ as a reliable stability margin for high order systems [9], [17], [18].

(2) More than one sensitivity peaks could be identified in high order systems (for example, in the design of resonance controllers, the Nyquist trajectory can lie close to the critical point for many different frequencies [17]). Therefore, in the context of a complex power system, the approximation given by (4a) or (4b) can be evaluated at different points of the Nyquist trajectory (i.e., at different critical frequencies).

(3) In principle, (4a) and (4b) seem redundant in the sense that both estimate $\sigma_{d}$. However, from a practical/datadriven point of view, it should be noted that, implicitly, there is an accuracy issue related to the finite number of point. More specifically, the denominators of (4a) and (4b) include differentiator terms, which implies a frequency resolution issue. Then, the criterion to choose between (4a) and (4b) is to use the one which a higher magnitude in the denominator.

\section{Simulation Results}

Fig. 6 depicts a simple MVDC power system employed for the analysis, whereas Table I shows its main parameters. It is inspired by a dc distribution that considers bidirectional power converters for both source and load sides: the source converter regulates the bus voltage at the point $P_{\mathrm{ev}}$ and the

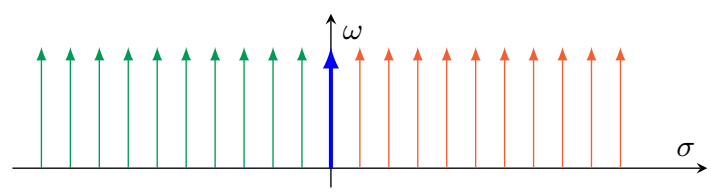

(a)

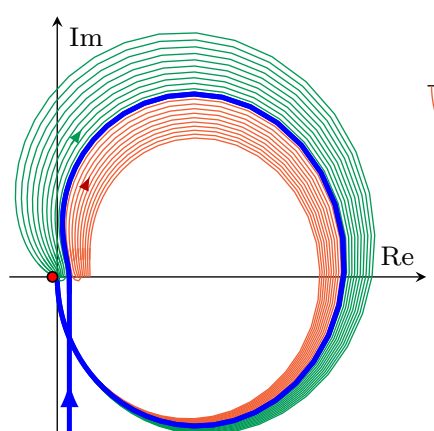

(b)

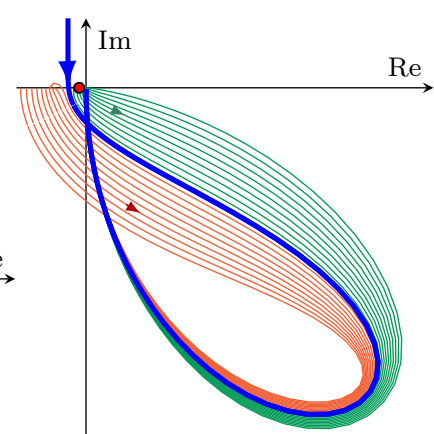

(c)
Fig. 4. Verification of the Nyquist stability criterion in the $G$-plane [arbitrary $G(s)$ are employed in this example]. (a) S-plane mapping: green curves corresponds to stable solutions $(\sigma<0)$; blue curves correspond to the Nyquist contour $(\sigma=0)$ and red curves corresponds to unstable solutions $(\sigma>0)$. (b) $G$-plane mapping for a stable system without RHP defined as $G(s)=\frac{(s+1)}{s(0.0073 s+0.459)} \frac{(0.1 s+1)^{2}}{(0.01 s+1)^{2}}$ : all the solutions that reach the critical point are stable (green). (c) $G$-plane mapping for a stable system with one RHP defined by $G(s)=\frac{(s+1)}{s(0.0073 s-0.459)} \frac{(0.1 s+1)^{2}}{(0.01 s+1)^{2}}$ : all the solutions that reach the critical point are stable (green).

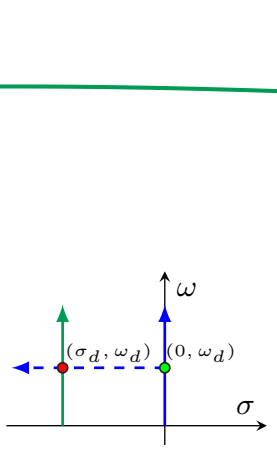

(a)

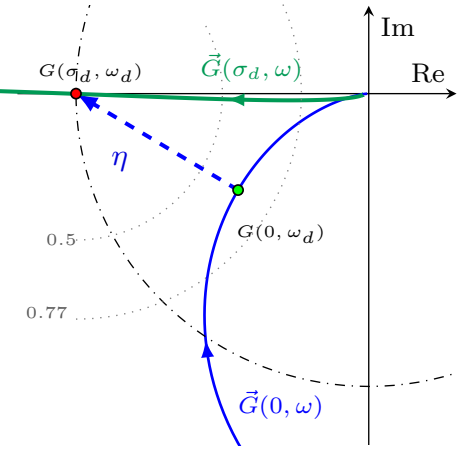

(b)
Fig. 5. Proposed estimation method (blue-dashed line). (a) S-plane theoretical contours: (blue) $0+j \omega$ trajectory, (green) $\sigma_{d}+j \omega$ trajectory, (blue-dashed) linear mapping assumption; (b) G-plane mapping (blue) $\vec{G}(0, \omega)$, (green) $\vec{G}\left(\sigma_{d}, \omega\right)$, (blue-dashed) ideal behaviour of the proposed approximation.

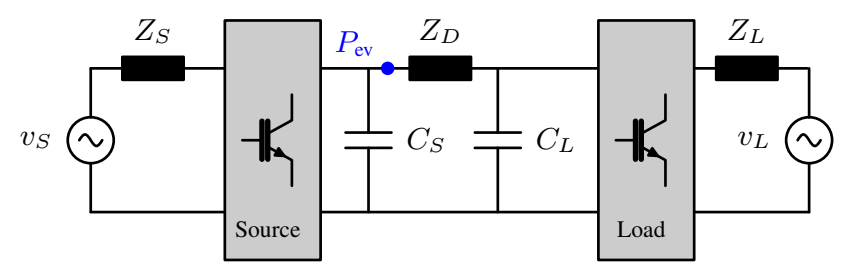

Fig. 6. Illustrative power circuit of a MVDC distribution system.

load inverter acts as a constant power load [19]. A ThreeLevel Neutral Point Clamped topology has been considered for both load and source converters. The controller bandwidths, which are dependent on the converters switching frequency, 
TABLE I

SiMULATION VALUES

\begin{tabular}{cc}
\hline \multicolumn{2}{c}{ MVDC circuit } \\
\hline Rated Power & $P=20 \mathrm{MW}$ \\
\hline DC voltage reference & $V_{C_{S}}^{*}=10 \mathrm{kV}$ \\
\hline AC voltage (both sides) & $v_{S}^{r m s}=v_{L}^{r m s}=6.6 \mathrm{kV}$ \\
\hline AC frequency (both sides) & $\omega_{1}=2 \pi 50 \mathrm{rad} / \mathrm{s}$ \\
\hline AC output impedances & $Z_{S}=Z_{L}=0.01+j 0.1 \mathrm{p} . \mathrm{u}$. \\
\hline DC capacitances & $Z_{C_{S}}=Z_{C_{L}}=-j 0.16 \mathrm{p} . \mathrm{u}$. \\
\hline Distribution impedance (cable effects) & $Z_{D}=0.001+j 0.5 \mathrm{p} . \mathrm{u}$. \\
\hline Control Parameters \\
\hline Sampling Frequency \\
\hline Inner loops bandwidth & $f_{s}=2 \mathrm{kHz}$ \\
\hline Outer loops bandwidth & $\alpha_{i}=880 \mathrm{rad} / \mathrm{s}$ \\
\hline
\end{tabular}

have been selected considering commercial devices suitable for MVDC systems [12]. The distribution line is represented by an inductive/resistive line $Z_{D}(\omega)$, which models long cable effects. The controller model has been implemented in Matlab/Simulink and the circuit components using the PLECS toolbox. $Z_{i}(\omega)$ and $Y_{o}(\omega)$ data are obtained by programming a frequency sweep in the perturbation signal [20], for a set of 200 frequencies logarithmically equally spaced from $1 \mathrm{~Hz}$ to $2 \mathrm{kHz}$. In the case of $Y_{o}(\omega)$ measurement, the perturbation is superimposed to the nominal voltage $V_{d c}$. Analogously, for the $Z_{i k}(\omega)$ measurement, the perturbation current is in parallel to a constant current consumption (rated value).

The key results for the ideal MVDC system are depicted in Fig. 7 and summarized in Table II. Fig. 7(a) represents $Z_{i}(\omega)$ and $Y_{o}(\omega)$ when $Z_{D}(\omega)$ is negligible. The source impedance $Z_{i}(\omega)$ is inductive at the low frequency range and capacitive in the high frequency range, and presents a wide and smooth magnitude peak at $5 \mathrm{~Hz}$, which has been found to be highly dependent on the source converter controller. In the case of the load admittance, it should be noted that $Y_{o}(\omega)$ is defined by a negative resistive behaviour in the low frequency range $\left(\angle Y_{o}(\omega)=180 \mathrm{deg}\right)$, as expected from a CPL [5], [6], [21], [22]. At the high frequency range, where the control action does not have an effect, $Y_{o}(\omega)$ is inductive and has a low magnitude. Fig. 7(b) shows the corresponding Nyquist diagram and the conformal mapping evaluation point. Table II shows the estimation of the dominant eigenvalue. For the sake of comparison with the frequency-domain results, Figs. 7(c)-7(d) show different time responses, which have been obtained by causing different transients in the circuit. These figures include an estimation of the settling time (within a $2 \%$ of the final value), which unfortunately is impaired by the presence of switching components. The decaying rate of the dominant eigenvalue is roughly estimated using the expression $\sigma_{d} \approx 3.9 / t_{s}$ [16]. From the observed $\sigma_{d}$ (time-domain results), it can be concluded that the conformal mapping predicts the main response with a good accuracy.

The simulation procedure is repeated with the consideration of a inductive cable impedance $Z_{D}(\omega)$ (see Table I), which

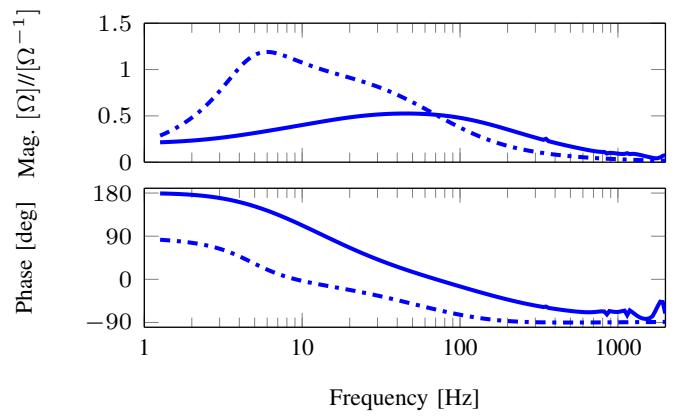

(a)
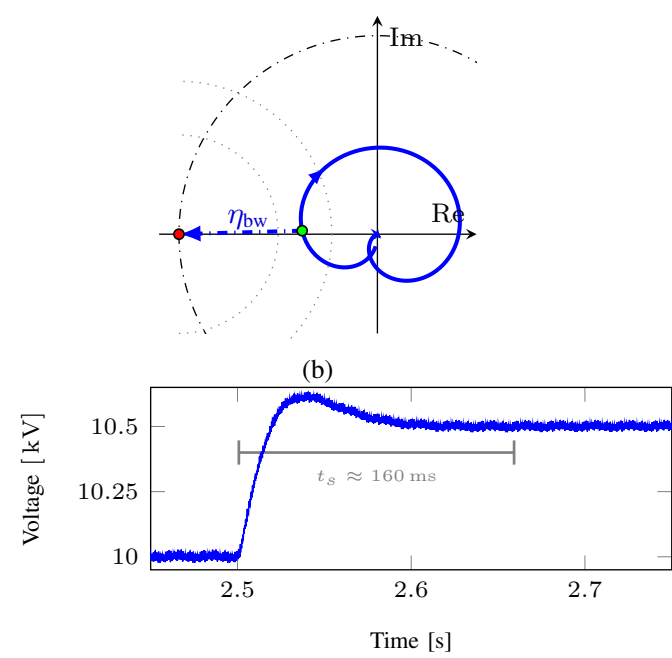

(c)

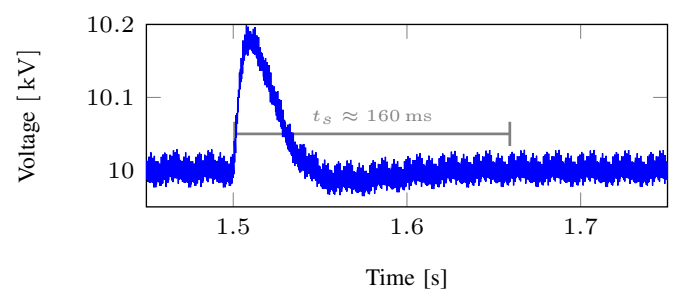

(d)

Fig. 7. Analysis and results for the ideal MVDC system. (a) $Z_{i}(\omega)$ (dashdotted) and $Y_{o}(\omega)$ (solid); (b) detail of $G(\omega)$ polar plot (Nyquist diagram); (c) $V_{d c}^{*}$ step change $\left(\Delta V_{d c}=500 \mathrm{~V}\right)$; (d) $200 \mathrm{~A}$ ideal current step change at dc bus.

TABLE II

SUMMARY OF RESULTS FOR THE IDEAL MVDC SYSTEM

\begin{tabular}{c}
\hline Conformal Mapping \\
\hline$\frac{p_{d}=-27.01 \pm j 33.52 \mathrm{rad} / \mathrm{s}\left(\eta_{\mathrm{bw}}=0.62\right)}{\text { Time-domain simulation }}$ \\
\hline$\sigma_{d} \approx 3.9 / t_{s}=-24.37 \mathrm{rad} / \mathrm{s},\left(\omega_{d}\right.$ unobservable $)$ \\
\hline
\end{tabular}

also splits the total capacitance in two parts and introduces an LC resonance in the dc circuit. Fig. 8(a) shows both $Z_{i}(\omega)$ and $Y_{o}(\omega)$ as measured in $P_{\mathrm{ev}}$. Fig. 8(b) represents the Nyquist trajectory. Two sensitivity peaks are considered, i.e., $\eta_{\text {bw }}$ due to closed loop bandwidth and $\eta_{\text {res }}$ due to the 
TABLE III

SUMMARY OF RESULTS FOR THE REALISTIC MVDC SYSTEM

\begin{tabular}{c}
\hline Conformal Mapping at $P_{\mathrm{ev}}$ \\
\hline$p_{d}=-14.94 \pm j 32.30 \mathrm{rad} / \mathrm{s}\left(\eta_{\mathrm{bw}}=0.52\right)$ \\
\hline$p_{d}^{\mathrm{res}}=-190.89 \pm j 400.59 \mathrm{rad} / \mathrm{s}\left(\eta_{\mathrm{res}}=0.66\right)$ \\
\hline Time-domain simulations \\
\hline$\sigma_{d}$ little affected by $Z_{D},\left(\omega_{d}\right.$ unobservable $)$ \\
\hline$\sigma_{d}^{\mathrm{res}}<-1 \mathrm{rad} / \mathrm{s}, \omega_{d}^{\mathrm{res}} \approx 232 \mathrm{rad} / \mathrm{s}$, attenuated \\
\hline
\end{tabular}

electric resonance in $Y_{o}(\omega)$; it should also be noted that the resonance also gives rise to a wide excursion of the Nyquist trajectory. Figs. 8(c)-8(d) show time domain responses in the presence of transients. Despite the resonance effect, the identification of the main response remains accurate, since $Z_{D}(\omega)$ effect in the low frequency range is quite negligible. However, the real part of the eigenvalues associated to $\omega_{d}^{\text {res }}$ is not identified with accuracy. The reason for the discrepancy is the fact that these eigenvalues are highly attenuated, i.e., their residue is much lower than 1 . In the frequency domain, attenuated responses are associated with open loop pole-zero cancellations in sensitivity transfer functions, and hence with a reduced observability of such a response [18].

\section{CONCLUSIONS}

Impedance stability modeling is a methodology suitable for assessing the dynamics of ac/dc power systems. As a contribution to this field, this paper properly provides new frequency-domain data-driven approach [i.e., $Z_{i}(\omega)$ and $Y_{o}(\omega)$ are obtained by small-signal perturbation] for the calculation of the system main responses. More specifically, analytic expressions for the calculation of the power system dominant eigenvalues is derived from an insightful interpretation of the Nyquist trajectory and its conformal mapping property. Subsequently, a study case, inspired on a MVDC distribution, has been developed and discussed. Finally, as a summary of practical outcomes is provided in the following.

(1) A good estimation of the dominant eigenvalues associated to load/source closed-loop interactions is achieved. The source/load interactions are usually in a low frequency range.

(2) The presence of attenuated oscillatory responses can be identified by the large excursions of the Nyquist trajectories. These take place in a relatively high frequency region, in which the closed-loop action is weak, and are associated with electrical resonances in the circuit.

(3) Due to a low observability condition (i.e., a zero-pole cancellation), a highly accurate estimation of decay rate and amplitude of responses associated with electric resonances is infeasible (by data-driven frequency domain methods).

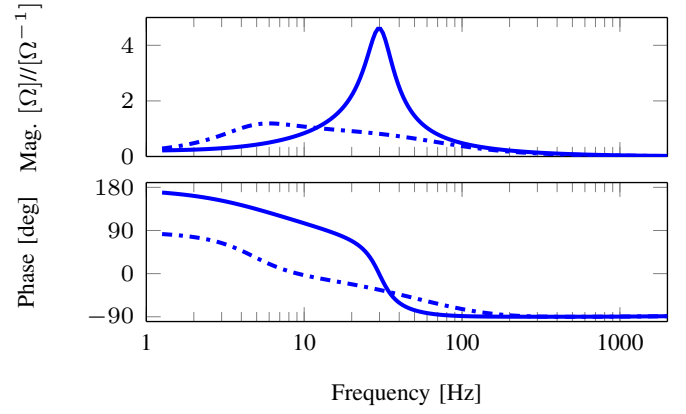

(a)

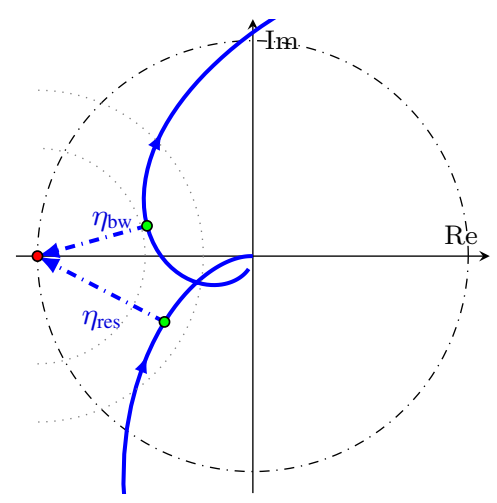

(b)

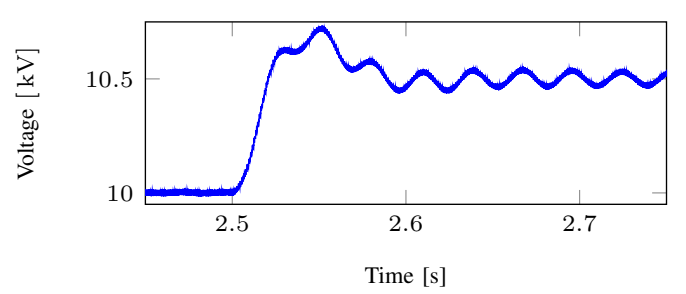

(c)

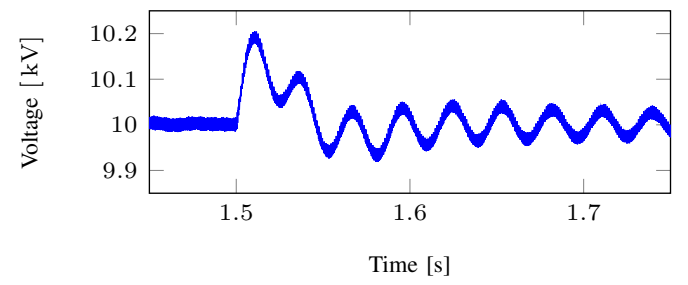

(d)

Fig. 8. Analysis and results for the ideal MVDC system. (a) $Z_{i}(\omega)$ (dashdotted) and $Y_{o}(\omega)$ (solid); (b) $G(\omega)$ Nyquist diagram; (c) $V_{d c}^{*}$ step change $\left(\Delta V_{d c}=500 V\right)$; (d) $200 \mathrm{~A}$ ideal current step change at the load. 


\section{REFERENCES}

[1] N. Hogan, "Impedance control: An approach to manipulation: Part I - theory," Journal of Dynamic Systems, Measurement, and Control, vol. 107 , no. 1 , p. 1,1985 .

[2] J. E. Colgate and N. Hogan, "Robust control of dynamically interacting systems," International journal of Control, vol. 48, no. 1, pp. 65-88, 1988.

[3] F. D. Freijedo, E. Rodriguez-Diaz, M. S. Golsorkhi, J. C. Vasquez, and J. M. Guerrero, "A root-locus design methodology derived from the impedance/admittance stability formulation and its application for LCL grid-connected converters in wind turbines," IEEE Trans. Power Electron., vol. 32, no. 10, pp. 8218-8228, Oct. 2016.

[4] J. Flower and C. Hodge, "Stability and transient-behavioural assessment of power-electronics-based dc-distribution systems: Part 1: The rootlocus technique," Journal of Marine Engineering \& Technology, vol. 3, no. 2, pp. 13-21, 2004.

[5] A. Riccobono and E. Santi, "Comprehensive review of stability criteria for DC power distribution systems," IEEE Trans. Ind. Appl., vol. 50, no. 5, pp. 3525-3535, Sep. 2014.

[6] T. Dragicevic, X. Lu, J. C. Vasquez, and J. M. Guerrero, "DC microgrids - part i: A review of control strategies and stabilization techniques,' IEEE Trans. Power Electron., vol. 31, no. 7, pp. 4876-4891, Jul. 2016

[7] M. Bradt, B. Badrzadeh, E. Camm, D. Mueller, J. Schoene, T. Siebert, T. Smith, M. Starke, and R. Walling, "Harmonics and resonance issues in wind power plants," in Power and Energy Society General Meeting, 2011 IEEE, pp. 1-8, 2011.

[8] J. L. Agorreta, M. Borrega, J. Lopez, and L. Marroyo, "Modeling and control of -paralleled grid-connected inverters with LCL filter coupled due to grid impedance in PV plants," IEEE Trans. Power Electron., vol. 26, no. 3, pp. 770-785, Mar. 2011.

[9] H. Kocewiak, J. Hjerrild, and C. Bak, "Wind turbine converter control interaction with complex wind farm systems," IET Renewable Power Generation, vol. 7, no. 4, pp. 380-389, 2013.

[10] L. Sainz, L. Monjo, J. Pedra, M. Cheah-Mane, J. Liang, and O. GomisBellmunt, "Effect of wind turbine converter control on wind power plan harmonic response and resonances," IET Electric Power Applications, vol. early access, 2017.

[11] Z. Liu, G. Zhang, and Y. Liao, "Stability research of high-speed railway emus and traction network cascade system considering impedance matching," IEEE Trans. Ind. Appl., vol. 52, no. 5, pp. 4315-4326, Sep. 2016.

[12] U. Javaid, F. D. Freijedo, D. Dujic, and W. van der Merwe, "Dynamic assessment of source-load interactions in marine MVDC distribution," IEEE Trans. Ind. Electron., vol. 64, no. 6, pp. 4372-4381, Jun. 2017.

[13] IEEE Recommended Practice for $1 \mathrm{kV}$ to $35 \mathrm{kV}$ Medium-Voltage DC Power Systems on Ships, IEEE Std 1709-2010 Std., Nov. 2010.

[14] M. R. Spiegel, Complex variables. Schaum, 1964.

[15] K. Astrom and T. Hagglund, PID Controllers: Theory, Design and Tuning, Second Edition. Instrument Society of America, 1995.

[16] R. C. Dorf and R. H. Bishop, Modern Control Systems. Prentice Hall, 2007.

[17] A. G. Yepes, F. D. Freijedo, O. Lopez, and J. Doval-Gandoy, “Analysis and design of resonant current controllers for voltage-source converters by means of nyquist diagrams and sensitivity function," IEEE Trans. Ind. Electron., vol. 58, no. 11, pp. 5231-5250, Nov. 2011

[18] G. C. Goodwin, S. F. Graebe, and M. E. Salgado, Control System Design. Prentice Hall, 2000.

[19] A. Yazdani and R. Iravani, "Dynamic model and control of the NPCbased back-to-back HVDC system," IEEE Trans. Power Del., vol. 21, no. 1, pp. 414-424, Jan. 2006.

[20] J. Sun, "Input impedance analysis of single-phase pfc converters," IEEE Transactions on Power Electronics, vol. 20, no. 2, pp. 308-314, Mar. 2005.

[21] S. D. Sudhoff, S. F. Glover, P. T. Lamm, D. H. Schmucker, and D. E. Delisle, "Admittance space stability analysis of power electronic systems," IEEE Trans. Aerosp. Electron. Syst., vol. 36, no. 3, pp. 965 973, Jul. 2000.

[22] A. Emadi, "Modeling and analysis of multiconverter DC power electronic systems using the generalized state-space averaging method," IEEE Trans. Ind. Electron., vol. 51, no. 3, pp. 661-668, Jun. 2004. 